who have previously suffered from liver complaints, any prolonged irritation of this nature may act most prejudicially.

'íhe absorption of mercury through the skin and mucous membrane of the nose, mouth, and throat, affords, in such instances, an admirable mode of producing the specific action of the medicine without communicating any additional irritation to any of the abdominal organs previously affected.

The symptoms of constitutional syphilis are sometimes masked by the coexistence of other diseases in tropical clinates; and, at other times, they do not there appear to develope themselves as they would in more temperate regions. Some of the most protracted cases of syphilis that have fallen under my notice, have aiforded instances of the secondary forms of the disease appearing when the patients have been suffering from diarrhœa, or from some form of fever, and when they have, consequently, not been subject to any sustained specific treatment. In some of these cases, the character of the eruption ${ }^{*}$ has long remained unrecognised, and the disease has continued to reappear for years in succession, always being attributed to the depressed state of the patient's health.

As soon as the real nature of the affection has been ascertained after a return to England, such patients have not only been relieved of their eruptions by the judicious use of the calomel bath, but have, at the same time, regained their general health.

Such cases as the preceding afford the best practical answer to those who, on the one hand, maintain that syphilis can be cured without mercury; and to those, on the other hand, who believe that syphilis can be satisfactorily treated by mercury administered internally.

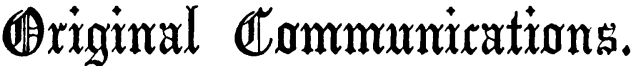

\section{ON THE VALUE OF OIL OF MALE-FERN AS A REMEDY FOR TAPE-WORM, AND ON THE MODE OF ADMINISTERING IT : WITH CASES.}

By J. D. Rendue, M.D., Medical Officer of the Government Convict Prison, Brixton, Surrey.

The report of Dr. Fleming, published in the British Medical Journal on January 16th of this year, on the value of the oil of male-fern as a remedy in tapeworm, has induced me to publish the following cases, all of which were successfully treated with this remedy.

The preparation of the patients by abstinence from solid food, and the mode of administering the oil, were somewhat different from that of the cases given in Dr. Fleming's report. I have, however, in every case of tape-worm which I have treated for the last few years, invariably given the remedy in ques tion, in the way I am about to describe; and the result in every instance, has been the rapid expulsion of the worm after a single dose of the drug.

The prisoners confined in the Brixton Prison are women under long sentences-penal servitude for three years and upwards. Cases of tape-worm are not of unfrequent occurrence among these prisoners during the early part of their sentences. The whole of the cases which follow have occurred since last July.
I will first give a short record of each case, and afterwards add the remarks I wish to make thereon.

CASE I. A. J., aged 22, a thin delicate woman, formerly resident in Liverpool, had been treated for tape-worm for many months before her admission to the Brixton Prison. Portions of the worm had been expelled, and the treatment gave temporary relief, but in a few weeks all the unfavourable symptoms returned. In July 1863, I ordered one dose of the oil of male-fern. A worm six yards and a quarter long was passed, since which she has never complained.

CASE II. S. S., aged 26, a strong and stout woman, formerly resident at Wakefield, had suffered from tapeworm for nine or ten years, for which she had been repeatedly under treatment, taking various remedies, including castor-oil and turpentine. Tape-worms, she stated, had frequently been passed after taking the remedies mentioned, but in two or three months there had always been a relapse. In August, after one dose of the oil of male-fern, a worm four yards and a half long was passed, and from that time she has been quite well.

CASE III is particularly important and interesting. A. B., aged 22, a healthy-looking strong woman, whose former place of residence was Manchester, was brought to the infirmary from her ward on August 27th, 1863, apparently in an epileptic fit. There had been no previous knowledge or suspicion of the existence of tape-worm; but, in an evacuation from the bowels consequent on the action of a black draught, a single joint of a tape-worm was discovered. The cause of the fit was thereby suspected, and the treatment directed for the expulsion of a worm. After a single dose of the oil of male-fern, a worm four yards in length was passed. This patient had suffered from occasional fits since the age of eleven years, but there had not been, as I before stated, any suspicion of the existence of tape-worm. I have repeatedly seen this woman since August, and up to this time (March 8 th), she has been altogether free from the fits she formerly suffered, and her general health has been good. During the first week of October, she thought that one or two joints of a small worm had on one occasion been passed from the bowels; I therefore at that time, subjected her a second time to treatment, and a thin narrow tape-worm, four inches in length, was passed after the usual dose of the malefern.

In every case of tape-worm which I have treated for the last three years, the mode of preparing the patient, the dose of the male-fern, and the way in which it was given, have been the same; all of which I will now briefly describe. The patient is sent to the infirmary, and late in the evening on the day of admis. sion, the treatment is commenced by giving an ordinary two-ounce black draught; on the following day all solid food is forbidden, but an almost unlimited supply of beef-tea is allowed; even milk, which is said to be the favourite food of the worm, I purposely forbid. On the evening of this day, half an ounce of castor oil is administered; and early on the following morning, about thirty-four hours after taking the black draught, two fluid drachms of the oil of malefern are given, suspended in two ounces of thin mucilage of acacia. The result without an exception, in every case which I have thus treated, has been the expulsion of a dead worm within two hours after taking the remedy, and in one instance the worm was passed in fifteen minutes.

I have had no difficulty in getting the patients to submit to this mode of treatment, nor have they complained of hunger. In order, lowever, to enable them to bear without much discomfort the prolonged abstinence from solid food, the supply of good beef- 
tea has, as I have stated, not only been unlimited, but each patient has also been kept in bed during the whole of the treatment.

The principle of this mode of treatment is, evidently, simply that of emptying the stomach and small intestines by abstaining from all solid food, and by purging; and so, by thus thoroughly uncovering the worm, exposing it to the full and almost immediate action of the remedy.

I have never, in a single instance, given more than one dose of the drug before the parasite was expelled; nor has the two-drachm dose given as I have mentioned, caused vomiting or troublesome purging; and in every case the worm was passed dead, and generally in one unbroken piece. No medicine of any kind is given except that which $I$ have before mentioned; and, in a few hours after the worm is passed, the patients are restored to their ordinary diet.

Before I was in the habit of preparing patients by the previous purging and abstinence which $I$ have described, I generally found that the oil of male-fern failed as a remedy for the cases under consideration ; but since I have adopted the mode of treatment which I now make public, I can say that I have never known it fail; and I cannot but feel certain that the remedy in question, if given as I have mentioned, will invariably, first poison, and then quickly dislodge this troublesome parasite from the human body.

\section{CASE OF GLAUCOMA WITH ACUTE INFLAMMATION, 'TREA'TED BY IRIDECTOMY.}

\section{By J. W. Hulke, Esq.}

AT the present moment, when so many persons doubt the efficacy of Iridectomy in Glaucoma, the following case is peculiarly valuable, from the circumstances under which the operation was performed, and from the fact that the results, after an interval of more than two years, are stated by an independent observer.

A lady, aged about 50, mentally deranged, residing under the charge of a physician to an asylum situated about sixty miles from town, lost the sight of her right eye from a deeply seated affection attended with severe inflammation, which rapidly ran its course, in spite of a well directed treatment. A few months after this, her left eye was similarly attacked. Her anxiety lest she should lose this, her only eye, was extreme. She was now brought to town by the physician, with an attendant, and reached my house at noon, August 24th, 1861.

The right eye was in the fourth stage of glaucoma - the stage of degeneration. The left eye was in the second stage of " glaucoma with peracute inflammation". The globe was very hard $(\mathrm{T}+3)$. The pupil was dilated and motionless. The anterior chamber was very shallow. The cornea was hazy. The optic nerve and retinal vessels could not be distinguished with the ophthalmoscope ; owing to the cloudiness of the transparent media, the fundus returned merely a faint red glow. The sclerotic and conjunctiva were very red; the latter was very œdematous, and overlapped the cornea. The upper eyelid was œdematous. Acuteness of vision had rapidly decreased. A couple of days previously, she had been able to read, but now the figure of a person at a yard's distance appeared to her as a dim shadow. The field of vision was much contracted; but her extreme distress prevented an exact estimate of the extent of this diminution. She was suffering constant violent pain, with paroxysmal exacerbations. In her own words, "I feel as if my head were coming off'”
In this condition, I knew that immediate iridectomy alone could prevent the destruction of the eye and I proposed that she should remain in town a few days, when I hoped she might be able to travel home. She readily acceded to the proposal of iridectomy. But then a difficulty arose: the physician held himself responsible for the safe charge of his patient; and he could neither remain with her in town, nor leave her safely in lodgings with the attendant. Thus it was evident that she must either forego the only measure which could save her eye, or submit to it notwithstanding the disadvantages of a fatiguing journey shortly after. I strongly urged the operation, in spite of this unfavourable circumstance. At 1 o'clock, she was put under the influence of chloroform ; and I performed iridectomy, removing about one-seventh of the iris. She awoke after sleeping about an hour, and took a light meal. At 4 o'clock, she left town by rail, wearing a light compress upon the eye, which felt sore, but was free from the violent pain experienced before the operation. The swelling of the eyelids was less-perhaps, in part, from the pressure of the compress. A fortnight afterwards, I heard that " the pain has not returned; all redness is gone ; the eye is still weak ; sight is improved." In reply to an inquiry, the physician, under whose care she still continued, wrote me the following letter.

"November 13th, 1863.

"Mr DEAR SiR,-I am glad to be able to inform you that the operation on Mrs. - 's eye was a great success. Her vision is really good, as she can read No. 2 (pearl) on your test-type sheet with facility. She cannot manage No. 1 (brilliant); nor could many others of her age, without previous disease. She wears spectacles, as she has done some years. The pain before the operation was very great; it, however, ceased entirely and immediately; and she has had none since. (The eye, however, which had been lost when you saw her, is still the seat of occasional pain, at times rather severe.) The tension of the eyeball is now natural, the hardness having disappeared. It would look like an ordinary eye, except for the change in the figure of the pupil, which is elliptical, with the iris curiously thinned at one edge; and there is a small fleshy tubercle at the cicatrix, which nevertheless is no inconvenience to her. I regard the case as one of the most successful I ever saw in surgery. I know, by the rate at which she became blind with the other eye, how soon she would have been totally blind, but for your help; and this, too, is her own thought on the subject. It is the more interesting, as she is decidedly insane, of a very passionate temperament, and in delicate health. "Very sincerely yours,

The Highly Moral Journal. The Dublin Medical Press and the British Medical Journal appear to be the only journals determined to put down the gigantic and still increasing evil of "gratuitous services," for which the latter journal comes in for an immense amount of editorial abuse from the Medical Times of the last two weeks. "Is Medicine a Human Profession?" Its last "leader" on the subject was no doubt intended by the writer for the approaching "Spring Meetings" at Exeter Hall; but being short of an article it appears this week in the Medical Times and Gazette, and was intended to annihilate the British Medical, which journal, however, returns again to the attack on the "gratuitous" humbugging system now prevailing, and, undeterred by the Pecksniffian sermonising of any journal, produces proofs every week of the injurious effect of the system on the profession at large. (Dublin Medical Press.) 